\section{Fonction des suppresseurs de tumeur PALB2 et BRCA2 dans la réparation des cassures double- brin de I'ADN}

\section{Rémi Buisson, Jean-Yves Masson}

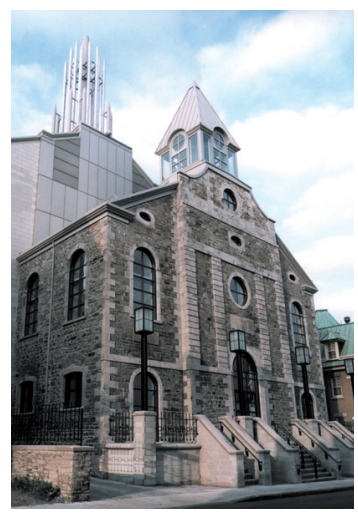

Genome stability laboratory, Laval university cancer research center, Hôtel-Dieu de Quebec research center (CHUQ), 9 McMahon, Québec, G1R 2J6, Canada. jean-yves.masson@crhdq. ulaval.ca

\section{Les mécanismes de réparation des cassures double-brin de l'ADN}

Les cellules de notre organisme subissent en permanence l'attaque d'agents endogènes ou exogènes qui entraînent des dizaines de milliers de lésions de I'ADN. Celles de type endogène sont provoquées par la cellule elle-même, et leur nombre a été estimé à plus de 100000 par jour [1]. Ces lésions sont créées, par exemple, lors de la réplication de l'ADN ou encore par l'action de métabolites réagissant avec la molécule d'ADN. Les lésions exogènes, quant à elles, sont produites par des sources physiques ou chimiques extérieures à la cellule. Les agents physiques, par exemple les radiations ionisantes, ou les agents chimiques génotoxiques, comme ceux qui sont utilisés lors des chimiothérapies anticancéreuses, créent une grande variété de lésions de l'ADN : modifications de bases, cassures simple-brin ou double-brin $[1,2]$. Hormis les cassures physiologiques programmées, lors de la méiose par exemple, les cassures double-brin sont les lésions de l'ADN les plus dangereuses pour les cellules, parce qu'elles menacent directement l'intégrité du génome et doivent être rapidement détectées, puis réparées. Les cellules ont développé pour ce faire plusieurs mécanismes de réparation.

Une cassure double-brin de l'ADN peut être réparée via quatre principaux mécanismes: le NHEJ (non-homologous end joining), le NHEJ alternatif (alt-NHEJ), la recombinaison homologue (RH), ou le SSA (single-strand annealing) (Figure 1 et Encadré) [1]. La cellule choisit l'un ou l'autre de ces mécanismes en fonction de la phase du cycle cellulaire et du type de cassure. Les NHEJ ou alt-NHEJ sont actifs durant tout le cycle cellulaire, alors que la RH ou le SSA sont actifs seulement à la fin de la phase $S$ et pendant la phase G2 [3]. Contrairement aux trois autres mécanismes, la RH est un mécanisme généralement fidèle de réparation de I'ADN. Dans cette revue, nous nous focaliserons plus particulièrement sur la réparation des cassures double-brin par recombinaison homologue.

\section{La recombinaison homologue}

La RH utilise la chromatide sœur présente après la réplication de l'ADN ou, lors de la méiose, un chromosome homologue comme une matrice comportant l'information génétique intacte permettant de réparer l'ADN brisé. Elle est subdivisée en trois étapes : la phase présynaptique, la phase synaptique et la phase postsynaptique (Figure IA). La recombinase RAD51, une protéine clé de ce processus, joue un rôle central au cours de ces trois phases. La phase présynaptique consiste 


\section{NHEJ (non-homologous end joining)}

Réparation par jonction d'extrémités non homologues permettant une religation directe des deux extrémités de l'ADN brisé (Figure 1B). Dans ce mécanisme, la cassure double-brin est détectée par le complexe Ku/DNA-PKcs (protein kinase, catalytic subunit), puis réparée par le complexe de ligation XLF-XRCC4 ( $X$-ray repair complementing defective repair in chinese hamster cells-4)-ADN ligase IV. Dans certains cas, l'exo-endonucléase Artémis est recrutée pour permettre la dégradation des extrémités de l'ADN afin de les rendre compatibles avec la ligation. Toutefois, cette activité est responsable de l'absence de fidélité du NHEJ puisqu'elle provoque la perte de plusieurs bases d'ADN.

\section{NHEJ alternatif (alt-NHEJ)}

Mécanisme indépendant du NHEJ initié par une nucléase, vraisemblablement par le complexe MRN (Mrell/Rad50/Nbsl)-CtIP, afin de générer des extrémités d'ADN simple-brin. Cette activité permet de découvrir des séquences de part et d'autre de la cassure comportant des microhomologies (2-4 nucléotides), dont l'hybridation permettrait la ligation de l'ADN (Figure 1E).

\section{SSA (single-strand annealing)}

Réparation par appariement entre molécules d'ADN simple-brin consistant en l'hybridation directe, grâce à la présence de séquences homologues (Figure IF), des ADN simple-brin générés lors de la résection des extrémités de la cassure. La protéine RPA (replication protein A) et la recombinase RAD52 sont au centre de ce mécanisme. Le SSA est principalement actif dans les régions du génome où les séquences sont dupliquées, ce qui représente environ $15 \%$ du génome humain. en la résection des extrémités de la cassure pour former un ADN 3'-protubérant. RAD51 se lie alors sur l'ADN simple-brin, autour duquel elle forme un filament nucléoprotéique. Durant la phase synaptique, RAD51 provoque l'invasion de l'ADN 3'-protubérant dans l'ADN doublebrin complémentaire de la chromatide sœur pour former une structure dite en D-loop (displacement loop ou boucle de déplacement). La phase postsynaptique consiste en l'extension de l'ADN envahissant par l'action d'une ADN polymérase afin de recopier l'information manquante $[4,5]$ (Figure 1).

Après l'étape d'extension, trois mécanismes différents peuvent être utilisés pour compléter la réparation : le DSBR, le SDSA ou le BIR. Dans la voie du DSBR (double-strand break repair), la seconde partie du brin brisé est capturée par les protéines RPA (replication protein $A$ ) et RAD52, puis associée au brin d'ADN déplacé, entraînant la formation de deux jonctions de Holliday ${ }^{1}$. La double jonction de Holliday (dHJ) est ensuite soit résolue, créant des produits d'ADN de type crossover ou non-crossover, soit dissoute pour donner naissance uniquement à des produits de type non-crossover (Figure IC). Dans la voie du SDSA (synthesis-dependent strand annealing), la D-loop est dissoute avant la formation de la $\mathrm{dHJ}$ pour s'hybrider avec l'ADN simple-brin

${ }^{1}$ Une jonction de Holliday est une jonction mobile entre quatre brins d'ADN. de la deuxième extrémité de la cassure. Ce mécanisme forme uniquement des produits d'ADN de type noncrossover (Figure ID). Dans la troisième voie appelée BIR (break-induced replication), la structure D-loop se convertit en fourche de réplication pour recopier le bras entier du chromosome (Figure IG). La voie du SDSA est prédominante dans la réparation par recombinaison homologue de l'ADN, tandis que la voie du BIR est la moins fréquente. Cette dernière est principalement utilisée lorsqu'il y a perte de la deuxième extrémité de la cassure, ou lors du processus de rallongement des télomères dans les cellules déficientes pour la télomérase [3-5].

\section{Régulation de la recombinaison homologue par les médiateurs BRCA2 et PALB2}

Lors de la phase présynaptique, l'ADN simple-brin formé à la suite de la résection de la cassure double-brin est dans un premier temps lié par la protéine RPA. La liaison de RPA protège l'ADN des attaques de nucléases et empêche la formation de structures secondaires ou la liaison de protéines inappropriées. RAD51 identifie ensuite un des segments d'ADN brisé, forme un filament nucléoprotéique, puis recherche dans le génome une séquence identique à celle qui a été endommagée [31] $\rightarrow$. Ce filament d'ADN- $\rightarrow$ Voir $m / s 2012$, RAD51 est l'élément central pour vol. 28, n 8-9, page 714 une réparation fidèle de la cassure double-brin. Pour ce faire, RAD51 s'oligomérise autour de l'ADN simple-brin pour former ce long filament hélicoïdal. Le filament a une conformation constante : un tour d'hélice du filament de RAD51 est constitué de six monomères, chacun liant trois bases d'ADN. L'association des monomères pour former ce nucléofilament requiert la liaison à l'ATP, dont l'hydrolyse toutefois n'est pas nécessaire [6]. La formation du filament de RAD51 nécessite le recrutement de cofacteurs afin notamment de déplacer RPA qui empêche la liaison de RAD51 sur l'ADN. Lors de la phase synaptique, ces mêmes cofacteurs stimulent son activité recombinase permettant ainsi l'étape d'invasion $[4,5]$. Ces cofacteurs sont des protéines appelées «médiateurs de la recombinaison homologue ». Chez l'humain, les protéines PALB2 (partner and localizer of BRCA2) et BRCA2 (breast cancer 2 protein) possèdent ces deux propriétés.

\section{$B R C A 2$ régule $R A D 51$ pour réparer les $C D B$}

Le gène BRCA2, localisé sur le chromosome 13q12-q13, a été identifié en 1995 comme étant un gène de susceptibilité au cancer du sein initialement, puis au cancer des ovaires, de la prostate ou encore du pancréas [7]. 

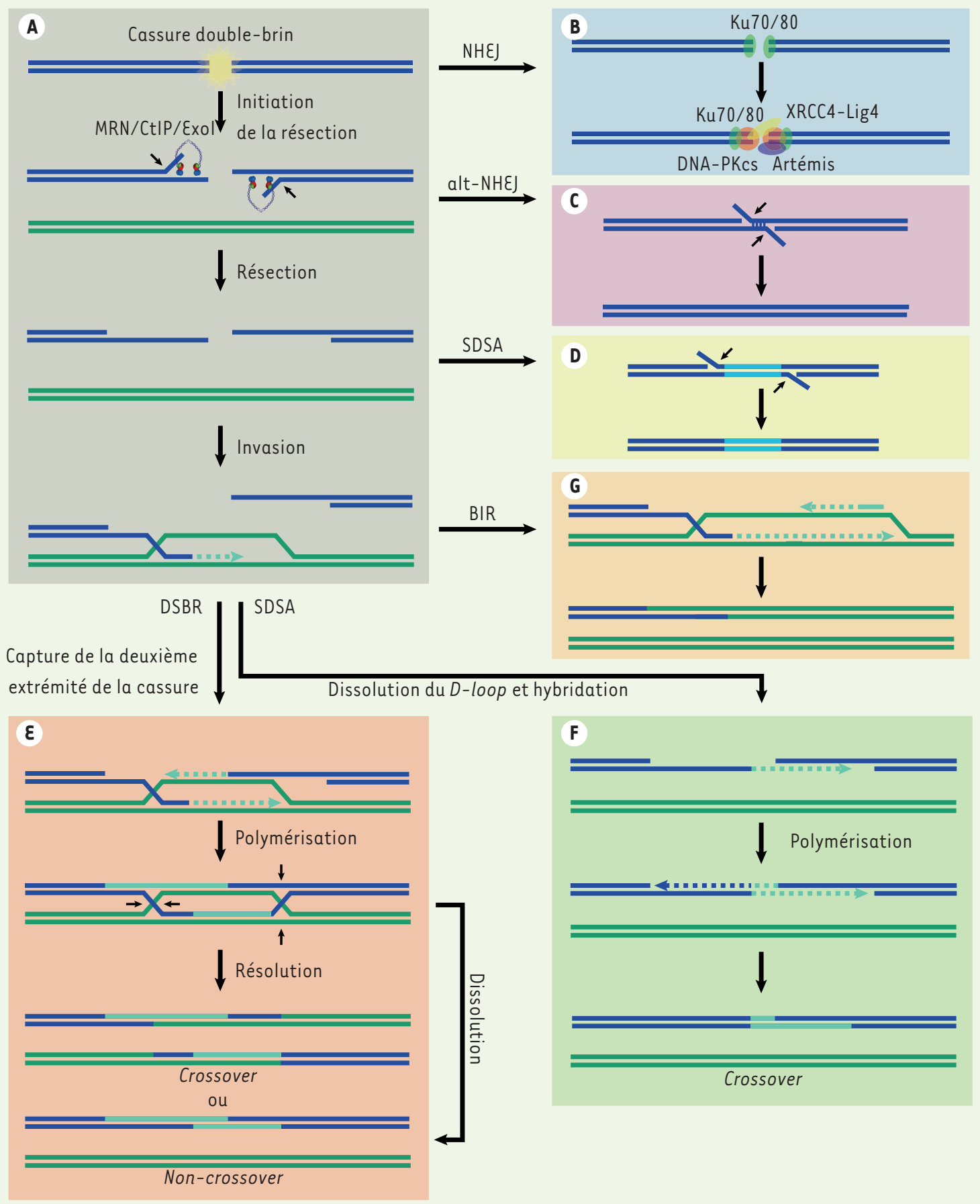

Figure 1. Mécanismes de réparation des cassures double-brin de l'ADN.

Entre 5 et $7 \%$ des cancers du sein sont provoqués par une mutation dans le gène BRCA2. Une personne possédant une mutation monoallélique de ce gène possède un risque de $50 \%$ de développer un cancer avant l'âge de 50 ans, et de $80 \%$ avant l'âge de 70 ans. Des mutations hypomorphes bialléliques dans BRCA2 entraînent le développement de l'anémie de Fanconi, une maladie génétique rare, causée par des mutations dans les gènes régulant la réparation des pontages interbrins de l'ADN [7].

La protéine humaine BRCA2 est une protéine de grande taille de 3418 acides aminés (384 kDa), qui est essentielle au fonctionnement de la recombinaison homologue $[3,7]$.

Cette dernière est diminuée de 80 à $90 \%$ en son absence. BRCA2 lie I'ADN simple-brin et, avec une plus faible affinité, I'ADN double-brin. La région de 


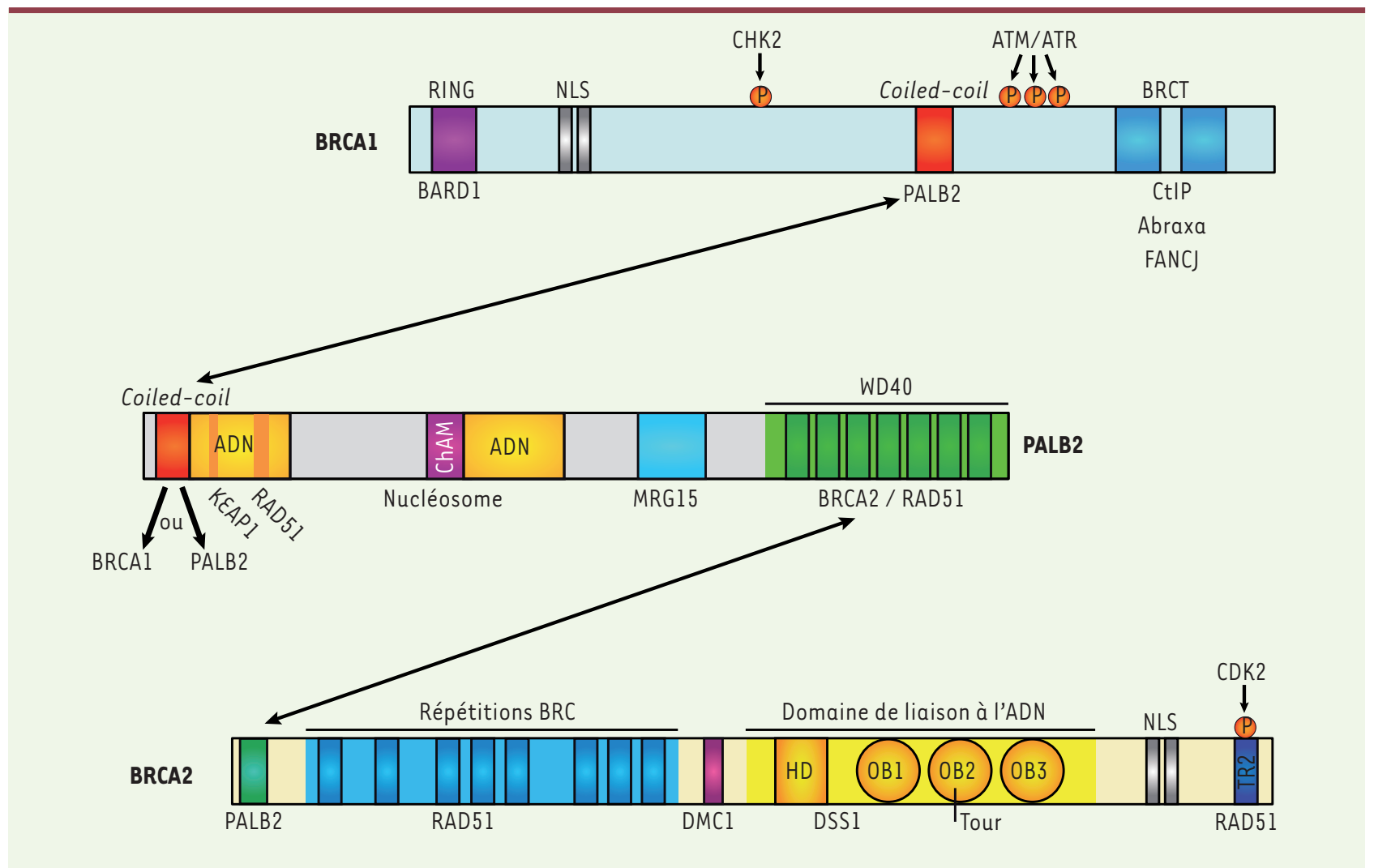

Figure 2. Organisation des domaines fonctionnels des protéines BRCA1, PALB2 et BRCA2. Les protéines interagissant avec BRCA1, PALB2 ou BRCA2 sont indiquées en dessous des domaines correspondants. Les flèches indiquent les interactions entre BRCAl, PALB2 et BRCA2. Les sites de phosphorylation sont indiqués par les cercles roses.

BRCA2 qui se lie à l'ADN est composée de cinq domaines [8]: un domaine en hélice (HD), trois domaines de liaison aux oligonucléotides (OB1, OB2 et OB3) qui lient I'ADN simple-brin, et un domaine en tour (tower) qui possède un sous-domaine en hélice-tour-hélice (HTH) responsable de l'activité de liaison à l'ADN double-brin (Figure 2). Les domaines $\mathrm{HD}$ et $\mathrm{OBl}$ sont également associés avec une autre protéine, appelée DSS1, importante pour la stabilité de BRCA2.

Le domaine de liaison à l'ADN de BRCA2 est important pour le recrutement de RAD51 aux cassures double-brin, qui est un des rôles majeurs de BRCA2. Par son interaction directe avec BRCA2 (une molécule de BRCA2 est capable de lier six molécules de RAD51 [9]), RAD51 est transportée dans le noyau de la cellule puis aux cassures doublebrin de I'ADN [10]. BRCA2 interagit avec RAD51 par deux domaines distincts : son domaine central composé de huit motifs BRC (environ 30 acides aminés) et son domaine carboxy-terminal. RAD51 lie préférentiellement les motifs $B R C 1,2,3$ et 4 , alors que les motifs $B R C$ $5,6,7$ et 8 lient plutôt le complexe RAD51-ADN [11]. Les fonctions exactes de ces répétitions de BRC sont encore imprécises. Dans les cellules, il a été montré que la surexpression de BRC4 empêche la localisation de RAD51 aux cassures double-brin [12]. In vitro, BRC3 et BRC4 détruisent le filament de RAD51 [10]. Néanmoins, cet effet ne s'observe que si la molarité de $B R C$ est très supérieure à celle de RAD51. À plus faible molarité, les motifs BRC3 ou BRC4 lient le filament de RAD51 et forment avec lui un complexe stable [13]. Récemment, deux autres études proposent que BRC4 ou BRCl-8 stimulent la formation du filament de RAD5l sur l'ADN simple-brin, ainsi que son activité d'échange de brin. L'interaction de BRC4 avec RAD51 diminue également l'activité d'hydrolyse de l'ATP de cette dernière (nécessaire au désassemblage du filament). À l'inverse, BRC4 empêche la formation du filament de RAD51 sur I'ADN double-brin [11]. Ce résultat est confirmé in vivo par une autre étude montrant que l'expression, dans des cellules déficientes en BRCA2, des motifs BRC3 ou BRC4 fusionnés au domaine de liaison à I'ADN de RPA (pour permettre leur localisation à la cassure indépendamment de BRCA2) restaure les foyers de RAD51, suggérant que BRC3 et BRC4 sont importants pour la stimulation du filament de RAD51 [14]. L'équilibre entre la stimulation de la formation et la déstabilisation du filament de RAD5l est donc probablement finement régulé par les motifs $B R C$ de BRCA2.

Le deuxième domaine d'interaction avec RAD5l est situé sur l'extrémité carboxy-terminale de BRCA2 et, plus exactement, au niveau d'un petit motif nommé TR2 qui est phosphorylé sur la sérine 3291 (Figure 2) [15]. 
A

$\mathrm{CDB}$

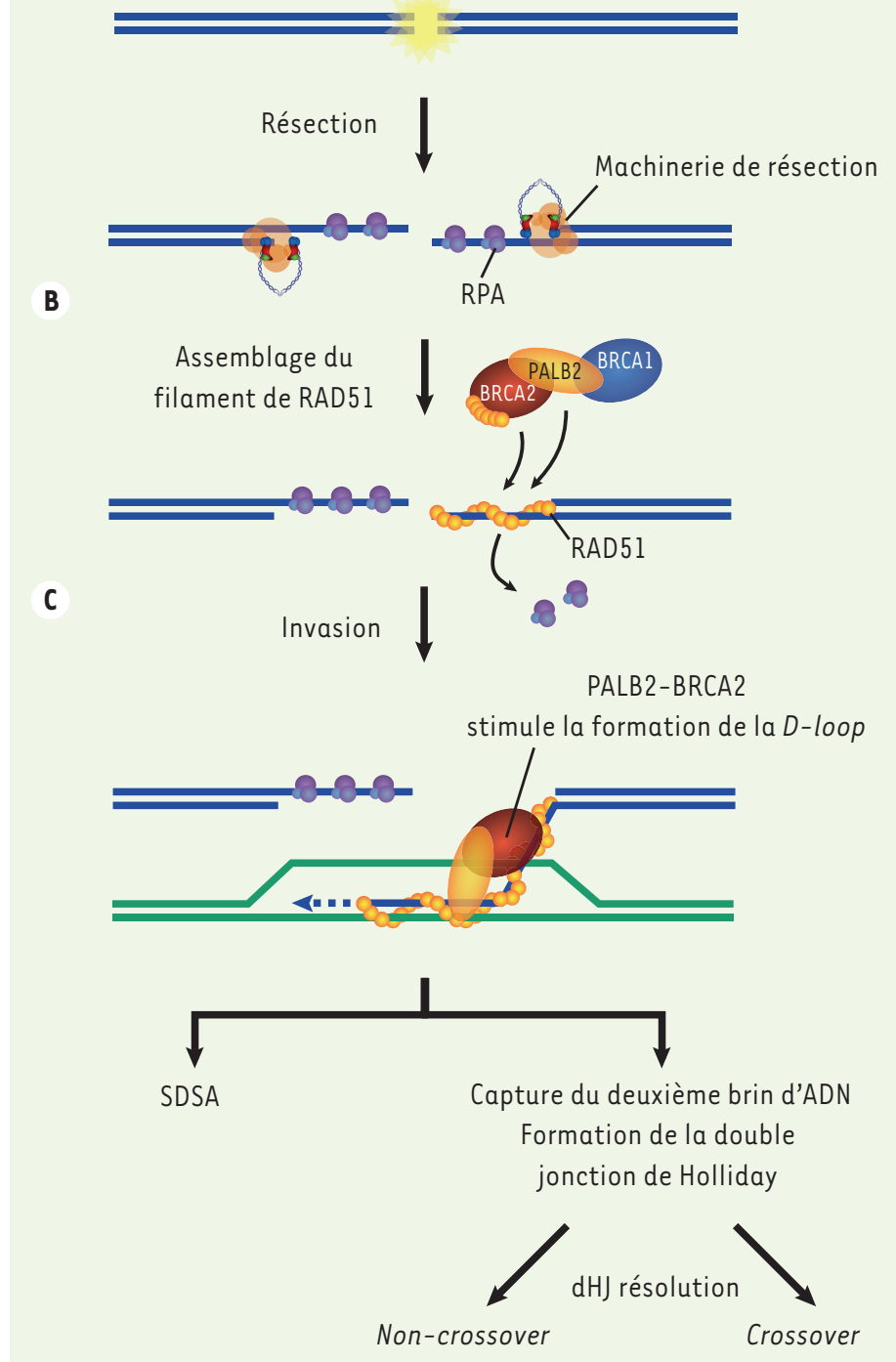

Figure 3. Fonction des médiateurs de la recombinaison homologue PALB2 et BRCA2. A. Résection de la cassure double-brin (CDB) par le complexe de résection pour former un ADN simple-brin protégé par RPA. B. Le complexe BRCAlPALB2-BRCA2 recrute RAD51 à la cassure double-brin, puis PALB2 et BRCA2 stimulent la formation de son filament autour de l'ADN simple-brin. C. PALB2 et BRCA2 stimulent l'étape d'invasion impliquant la RAD51. dHJ : double jonction de Holliday. SDSA : synthesis-dependent strand annealing.

La forme phosphorylée de TR2 est incapable de lier RAD51 mais, en présence de cassures double-brin dans la cellule, la sérine 3291 est déphosphorylée, ce qui provoque son interaction avec RAD51 [15] et la protection de son filament contre l'activité destructrice du motif BRC4 $[16,17]$. Une autre étude a montré que BRCA2 protège les brins d'ADN néosynthétisés lorsque la progression des fourches de réplication est perturbée. Le domaine TR2 joue un rôle important dans cette activité en protégeant l'ADN nouvellement formé de la dégradation par MRE1l (meiotic recombination 11 homolog 1) [18]. Toutes ces observations indiquent que BRCA2, par ses domaines BRC et TR2, contrôle la formation du filament de RAD51 pour la réparation des cassures double-brin ou le redémarrage des fourches de réplication, puis son désassemblage pour permettre la poursuite du cycle cellulaire [19].

\section{Rôle des interactions protéiques de PALB2}

et de sa dimérisation dans la réparation des cassures double-brin

La protéine PALB2 a été identifiée en 2006 en immunoprécipitant la protéine BRCA2 [20]. Le gène PALB2 est localisé sur le chromosome 16p12.2 et consiste en 13 exons qui codent pour une protéine de 1186 acides aminés $(131 \mathrm{kDa})$. Une mutation hétérozygote dans le gène PALB2 est à l'origine de prédispositions au cancer du sein et du pancréas, alors que des mutations homozygotes, comme pour le gène BRCA2, causent l'anémie de Fanconi [21].

PALB2 interagit avec BRCA2 via un domaine constitué de sept motifs WD40 situé en carboxy-terminal de la protéine. BRCA2, quant à elle, interagit avec PALB2 grâce à son domaine situé en amino-terminal. L'absence de PALB2 dans les cellules induit une perte de la localisation de BRCA2, et donc de RAD51, aux cassures double-brin, d'où son nom : partenaire localisateur de BRCA2 (parther and localizer of BRCA2) [20]. PALB2 lie également la protéine BRCAl via son domaine coiled-coil situé à son extrémité amino-terminal, et cette interaction est essentielle à sa propre localisation aux cassures doublebrin [22]. Ce domaine permet également la dimérisation de PALB2 [23]. Un troisième domaine, nommé ChAM (chromatin association motif), est quant à lui important pour l'association de PALB2 à la chromatine [24]. La fonction initialement attribuée à PALB2 était d'ancrer BRCA2 aux structures nucléaires, mais sa fonction biochimique exacte, en revanche, restait à élucider.

Des études biochimiques, conduites indépendamment dans notre laboratoire et celui du Pr. P. Sung, ont révélé une interaction directe entre PALB2 et RAD51, ainsi qu'une activité de liaison de PALB2 à l'ADN. PALB2 possède deux domaines de liaison à l'ADN situés en amino-terminal et au centre de la protéine (Figure 2), qui lient préférentiellement les structures d'ADN en forme de D-loop [25, 26]. Ceci suggère que PALB2 pourrait directement influencer l'activité de RAD51 dans la recombinaison homologue. À l'aide d'essais enzymatiques, il a été récemment démontré que PALB2, ainsi que BRCA2, (1) stimulent la formation du filament de RAD51 sur un ADN simple-brin protégé par la protéine RPA, et (2) stimulent l'échange des brins catalysé par le filament nucléoprotéique RAD51 [9, 25-28]. Ces activités font de PALB2 et BRCA2 des médiateurs de la recombinaison homologue (Figure 3 ). 


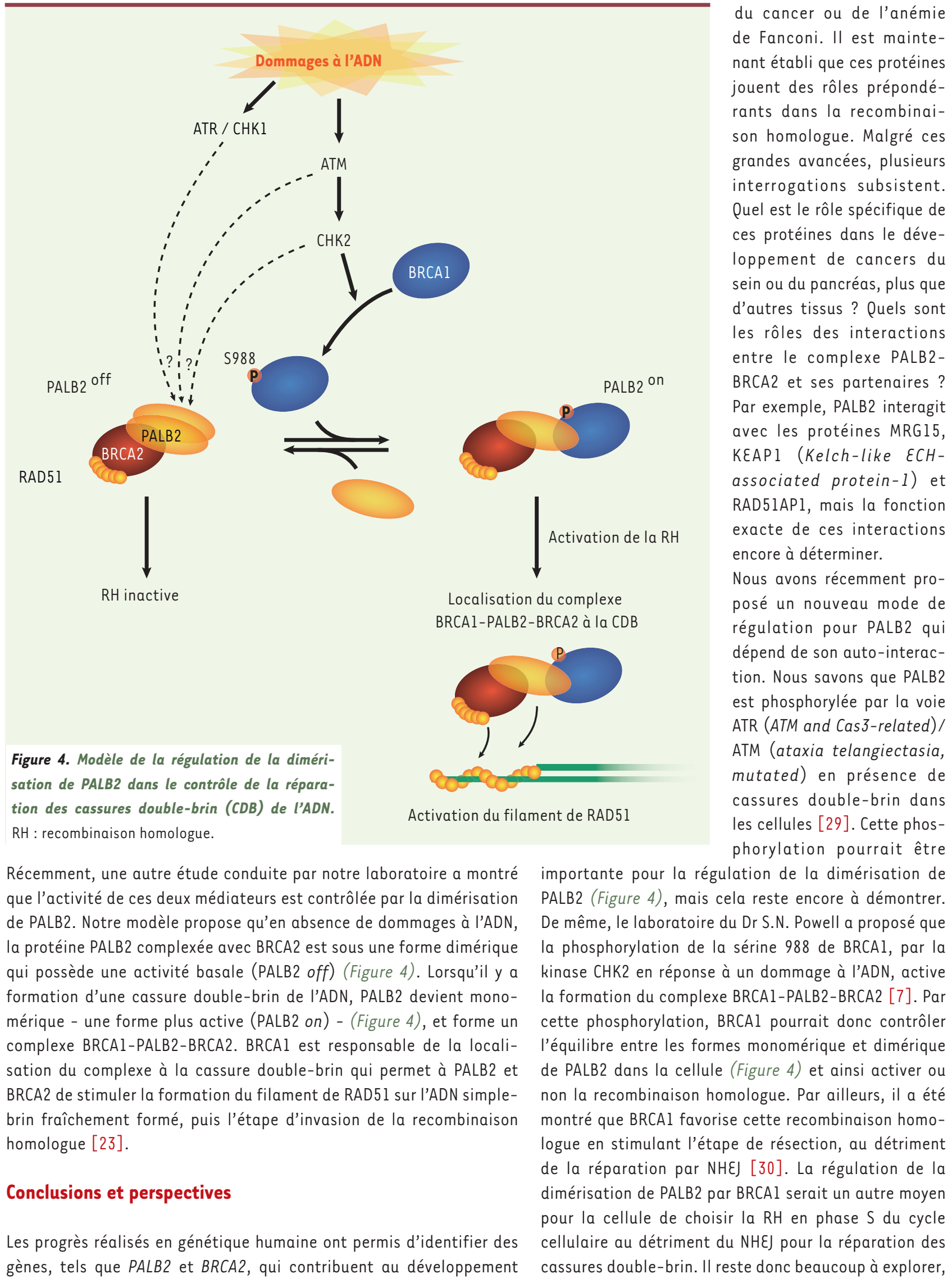


mais l'étude des médiateurs de la recombinaison homologue permettra de mieux comprendre les mécanismes de préservation de l'intégrité du génome et, ainsi, de prévenir les maladies humaines s'y rattachant.

\section{SUMMARY}

Functions of PALB2 and BRCA2 tumor suppressors in DNA double-strand break repair

Cancer is now the leading cause of mortality in France. It has been clearly demonstrated that mutations in the genetic information is the initiating event of cancer. DNA damage such as DNA double-strand breaks leads to genomic instability and cancer development. Cells can repair DNA double-strand breaks through several mechanisms. Nevertheless, only homologous recombination repair is faithful and repairs DNA without creating mutations. Here, we review the roles of PALB2 and BRCA2 in homologous recombination and genome stability. $\diamond$

\section{LIENS D'INTÉRÊT}

Les auteurs déclarent n'avoir aucun lien d'intérêt concernant les données publiées dans cet article.

\section{REMERCIEMENTS}

Les auteurs remercient Joris Pauty, Anthony Couturier, Eloise Dray, Patrick Sung, Bing Xia, Isabelle Brodeur, et le laboratoire Masson pour les discussions sur le sujet. Ce projet est soutenu par les IRSC. R. B. est boursier du FQRNT et J.-Y. M. est chercheur senior du FRSP.

\section{RéFÉRENCES}

1. Ciccia A, Elledge SJ. The DNA damage response: making it safe to play with knives. Mol Cell 2010 ; $40: 179-204$

2. Lord CJ, Ashworth A. The DNA damage response and cancer therapy. Nature $2012 ; 481: 287-94$.

3. Moynahan ME, Jasin M. Mitotic homologous recombination maintains genomic stability and suppresses tumorigenesis. Nat Rev Mol Cell Biol $2010 ; 11$ : 196-207.

4. San Filippo J, Sung P, Klein H. Mechanism of eukaryotic homologous recombination. Ann Rev Biochem $2008 ; 77: 229-57$.

5. Krejci L, Altmannova V, Spirek M, Zhao X. Homologous recombination and its regulation. Nucleic Acids Res $2012 ; 40: 5795-818$.

6. West SC. Molecular views of recombination proteins and their control. Nat Rev Mol Cell Biol 2003 ; $4: 435-45$.

7. Roy R, Chun J, Powell SN. BRCAl and BRCA2: different roles in a common pathway of genome protection. Nat Rev Cancer $2012 ; 12: 68-78$.

8. Yang $\mathrm{H}$, Jeffrey PD, Miller J, et al. BRCA2 function in DNA binding and recombination from a BRCA2-DSS1-ssDNA structure. Science 2002 ; 297 : 1837-48.

9. Liu J, Doty T, Gibson B, Heyer WD. Human BRCA2 protein promotes RAD51 filament formation on RPA-covered single-stranded DNA. Nat Struct Mol Biol $2010 ; 17$ : 1260-2.

10. Davies AA, Masson JY, Mcllwraith MJ, et al. Role of BRCA2 in control of the RAD51 recombination and DNA repair protein. Mol Cell $2001 ; 7: 273-82$

11. Carreira A, Kowalczykowski SC. Two classes of BRC repeats in BRCA2 promote RAD51 nucleoprotein filament function by distinct mechanisms. Proc Natl Acad Sci USA 2011 ; 108 : 10448-53.
12. Chen CF, Chen PL, Zhong $\mathrm{Q}$, et al. Expression of BRC repeats in breast cancer cells disrupts the BRCA2-Rad51 complex and leads to radiation hypersensitivity and loss of G(2)/M checkpoint control. J Biol Chem 1999 ; $274: 32931-5$.

13. Galkin VE, Esashi F, Yu X, et al. BRCA2 BRC motifs bind RAD51-DNA filaments. Proc Natl Acad Sci USA $2005 ; 102: 8537-42$.

14. Saeki $H$, Siaud N, Christ N, et al. Suppression of the DNA repair defects of BRCA2-deficient cells with heterologous protein fusions. Proc Natl Acad Sci USA $2006 ; 103: 8768-73$.

15. Esashi F, Christ N, Gannon J, et al. CDK-dependent phosphorylation of BRCA2 as a regulatory mechanism for recombinational repair. Nature $2005 ; 434$ : 598-604.

16. Esashi F, Galkin VE, $Y_{u} X$, et al. Stabilization of RAD51 nucleoprotein filaments by the C-terminal region of BRCA2. Nat Struct Mol Biol 2007 ; 14 : 468-74.

17. Davies OR, Pellegrini L. Interaction with the BRCA2 $C$ terminus protects RAD51-DNA filaments from disassembly by BRC repeats. Nat Struct Mol Biol $2007 ; 14: 475-83$

18. Schlacher K, Christ N, Siaud N, et al. Double-strand break repairindependent role for BRCA2 in blocking stalled replication fork degradation by MRE11. Cell $2011 ; 145: 529-42$.

19. Ayoub N, Rajendra $\varepsilon$, Su X, et al. The carboxyl terminus of Brca2 links the disassembly of Rad51 complexes to mitotic entry. Curr Biol 2009 ; 19 : 1075 85.

20. Xia B, Sheng $Q$, Nakanishi $K$, et al. Control of BRCA2 cellular and clinical functions by a nuclear partner, PALB2. Mol Cell $2006 ; 22$ : 719-29.

21. Tischkowitz M, Xia B. PALB2/FANCN: recombining cancer and Fanconi anemia. Cancer Res $2010 ; 70: 7353-9$.

22. Zhang $\mathrm{F}, \mathrm{Ma}$ J, Wu J, et al. PALB2 links BRCAl and BRCA2 in the DNA-damage response. Curr Biol $2009 ; 19: 524-9$.

23. Buisson R, Masson JY. PALB2 self-interaction controls homologous recombination. Nucleic Acids Res $2012 ; 40$ : 10312-23.

24. Bleuyard JY, Buisson R, Masson Jy, Esashi F. ChAM, a novel motif that mediates PALB2 intrinsic chromatin binding and facilitates DNA repair. EMBO Rep 2012 ; 13 : 135-41.

25. Buisson R, Dion-Cote AM, Coulombe Y, et al. Cooperation of breast cancer proteins PALB2 and piccolo BRCA2 in stimulating homologous recombination. Nat Struct Mol Biol 2010 ; 17 : 1247-54.

26. Dray $\varepsilon$, Etchin J, Wiese C, et al. Enhancement of RAD51 recombinase activity by the tumor suppressor PALB2. Nat Struct Mol Biol $2010 ; 17$ : 1255-9.

27. Jensen RB, Carreira A, Kowalczykowski SC. Purified human BRCA2 stimulates RAD51-mediated recombination. Nature $2010 ; 467: 678-83$.

28. Thorslund T, Mcllwraith MJ, Compton SA, et al. The breast cancer tumor suppressor BRCA2 promotes the specific targeting of RAD51 to singlestranded DNA. Nat Struct Mol Biol $2010 ; 17$ : 1263-5.

29. Matsuoka S, Ballif BA, Smogorzewska A, et al. ATM and ATR substrate analysis reveals extensive protein networks responsive to DNA damage. Science 2007 ; 316 : 1160-6.

30. Bunting SF, Callen $\varepsilon$, Wong N, et al. 53BPl inhibits homologous recombination in Brcal-deficient cells by blocking resection of DNA breaks. Cell $2010 ; 141: 243-54$.

31. Miné-Hattab J, Rothstein R. Réparation de l'ADN : comment trouver le bon partenaire? Med sci (Paris) $2012 ; 28$ : 714-6.

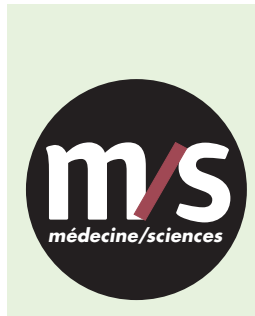

Tarifs d'abonnement $m / s-2013$

Abonnez-vous

à médecine/sciences
$>$ Grâce à $m / s$, vivez en direct les progrès des sciences biologiques et médicales

\section{TIRÉS À PART}

J.Y. Masson 